\title{
Objetos incómodos: el lugar de las "uma-lulik" en el Estado-Nación de TIMOR-LESTE
}

\author{
Alberto Fidalgo Castro ${ }^{1}$ \\ Universidade da Coruña, Ferrol, España
}

Este articulo tiene como objetivo contribuir al debate actual sobre la formación del Estado-Nación timorense, especificamente con respecto a las politicas culturales relacionadas con las uma-lulik (casas sagradas). Argumento que el proceso de estetización de la cultura timorense, en particular las intervenciones en materia de patrimonio, tiene como resultado la pérdida del sentido de la cultura en tanto práctica social viva. Esta reducción de la cultura a sus elementos más visibles responde a un uso cosmético de la misma. Mediante este proceso de resemantización, la cultura pierde su razón de ser y pasa a convertirse en fuente de tensiones y posibles conflictos.

Palabras clave: Timor-Leste, construcción nacional, sociedades de casas, políticas culturales, estetización

\section{INTRODUCCIÓN}

Este artículo tiene como intención contribuir al conocimiento del estado de la cuestión en el debate sobre la formación del Estado-Nación timorense, específicamente acerca de las políticas culturales relacionadas con las uma-lulik (casas sagradas). En él, retomo la postura presentada por Silva \& Simião (2012), quienes, tomando como punto de partida las variaciones sobre las prácticas matrimoniales, analizan los discursos que se generan en torno a ellas en tanto que estructuradoras de socialidad en el contexto urbano. Estos discursos encubren ideas sobre qué debería ser una nación y cuál es la manera de articular la cultura tradicional adat en la creación

1 Doctorando en Antropología del Departamento de Humanidades, Facultade de Humanidades. Investigador miembro del Arthropology Lab. E-mail: alberto.fidalgo.castro@udc.es

2 Adat es una palabra de origen árabe que se traduce habitualmente como "costumbre" (Cribb y Kahin 2004:6). Englobo dentro del término adat otra serie de conceptos que podrían resultar sinónimos en determinados contextos, como lisan (tradición) o kultura (cultura). He tratado este tema con mayor amplitud en otro lugar, véase 
del Estado-Nación de Timor-Leste. Tratan, asimismo, sobre el papel que tales prácticas juegan en la creación identitaria de la imaginación como nación. ${ }^{3}$

Después del referéndum de 1999, en el que Timor Oriental consiguió la independencia, la Organización de las Naciones Unidas (ONU) comenzaron a construir un estado que tenía en poca consideración la cultura local. ${ }^{4} \mathrm{La}$ devolución de la soberanía a los timorenses en 2002 supuso la primera ocasión en que, desde la llegada del colonialismo, los timorenses podían construir un estado a su voluntad. Así, se introdujo en la Constitución un artículo que establece: "O Estado reconhece e valoriza as normas e os usos costumeiros de Timor-Leste que não contrariem a Constituição e a legislação que trate especialmente do direito costumeiro" (RDTL 2002). La organización social tradicional quedaba reconocida de este modo, pero con una categoría de rango inferior a la del ordenamiento jurídico formal. Las llamadas a la necesidad de tener más en cuenta los elementos de la sociedad tradicional en la construcción del Estado-Nación timorense han sido foco de atención y debate desde diversos puntos de vista: la gobernabilidad (Hicks 2013; Magno y Coa 2012; Trinidade 2008); la justicia (Babo-Soares 2004, 2007; Kovar y Harrington 2013; Nixon 2012; Simião 2005, 2011, 2013; Yoder 2007) o la gestión de los recursos naturales (Do Amaral de Carvalho y Coreia 2011; D'Andrea, Silva, y Yoder 2003). Sin embargo, el escrutinio teórico y etnográfico al que se ha sometido la necesidad de articular la cultura tradicional y el aparato del Estado no ha tenido como contrapartida una plasmación clara en las políticas estatales, al menos de momento; algunos ejemplos son las dificultades para aprobar las conocidas como Leyes de la Tierra ${ }^{5}$ (vetadas por el presidente en 2012) o la incapacidad para articular la justicia consuetudinaria y el sistema formal.

La cultura ha sido clasificada, dentro del organigrama de los sucesivos gobiernos, bajo diversas carteras ministeriales: el Ministerio de Educación, Cultura, Juventud y Deporte (primer Gobierno Constitucional); el Ministerio de Educación y Cultura (segundo y tercer Gobierno Constitucional), el Ministerio de Educación (cuarto Gobierno Constitucional) y, finalmente, el Ministerio de Turismo (quinto -y actual- Gobierno Constitucional). En este tránsito se ha producido también un cambio en la denominación de la secretaría de estado correspondiente, que ha pasado de ser simplemente la Secretaría de Estado de la Cultura a llamarse Secretaría de Estado de Arte y Cultura. En este cambio se puede colegir una intencionalidad clara: la de asociar la cultura, de manera metonímica, a la esfera de lo artístico, lo patrimonial y lo turístico, es decir, en mi opinión, la puesta en marcha de un proceso de estetización de elementos muy visibles de la cultura tradicional que, resemantizados dentro de un contexto social, pierden su sentido político y práctico (a la vez que ganan otros sentidos), lo cual puede ser una fuente de conflictos. Sostengo, al igual que muchos otros autores (Bovensiepen 2014; Gárate Castro y Assís 2010; Hicks 2013; Silva y Simião 2012), que las uma-lulik son uno de estos elementos, acaso el más importante de ellos; pero intento demostrar cómo el proceso de puesta en valor patrimonial-artístico y turístico puede tener y tiene efectos adversos y no deseados tanto en el nivel local como en el nacional.

Fidalgo Castro (2012).

3 Leach (2012:240) ha escrito sobre la creciente importancia que se le otorga al papel que las costumbres (adat) juegan en la creación de la identidad timorense entre los jóvenes universitarios en un contexto urbano.

4 Kelly Silva, en un reciente libro (2012), ha revisado las prácticas de las Naciones Unidas en la construcción del Estado-Nación de Timor Oriental.

5 Las Leyes de la Tierra son las siguientes: Lei ba Rai (Regime Especial para a Definição da Titularidade dos Bens Imóveis), Dekretu Parlamentar 69/II; Lei Expropriasaun (Lei das Expropriações), Dekretu Parlamentar 70/II; Lei Fundu Finanseiru Imobiliariu (Fundo Financeiro Imobiliário), Dekretu Parlamentar 71/II (Anon 2012). 
Para ello he dividido el texto en tres partes. En la primera realizo una revisión del concepto de uma-lulik en la literatura antropológica, y menciono brevemente alguna de las maneras en que las diferentes políticas culturales de los poderes históricos (Portugal e Indonesia) han abordado la relación entre Estado y adat. En la segunda me aproximo al debate teórico acerca de la estetización de la cultura en el escenario de la cultura toraya, con el objetivo de señalar paralelismos entre las políticas culturales aplicadas entre los toraya y las que se aplican hoy en día en Timor Oriental. Finalmente presento tres pequeños estudios de caso en los que se analiza cómo la reducción de la cultura local a una mera expresión artística o patrimonial choca con la cultura puesta en práctica y cómo, irónicamente, las políticas cultuales han de enfrentarse a los usos políticos de la cultura.

\section{Las casas sagradas de Timor Oriental y su relación con el Estado portugués, el Estado indonesio y la República Democrática de Timor-Leste}

El estudio desde la antropología de las sociedades de casas tiene un importante corpus teórico y etnográfico, buena parte del cual se ha desarrollado en Indonesia Oriental, área en la que tradicionalmente se ha incluido a Timor Oriental. Mucho se ha escrito acerca de la casa como institución social desde que Lévi-Strauss propusiera, en La vía de las máscaras (1985 [1979]), que la société à maison era un tipo de organización social diferenciada: "estas casas, de las que Kroeber considera sólo la técnica de construcción y la función utilitaria [...] constituyen, de hecho, personas morales"(1985:149). Más allá de las críticas que se puedan realizar acerca de la validez de la casa como concepto analítico tal y como lo planteó Lévi-Strauss, y de su aplicación a todo el Sudeste Asiático ${ }^{6}$ (véanse, por ejemplo, Carsten y Hugh-Jones 1995; Fox 1987), hay que reconocer que abrió todo un camino en un campo de investigación que hasta aquel momento se circunscribía -salvando algunas excepciones- a los elementos más materiales y folclóricos de las casas. No puede negarse que Lévi-Strauss creó escuela, también en este aspecto. ${ }^{7}$

Ahora bien, ¿qué son las uma-lulik timorenses? Diversos registros coloniales utilizaron la palabra pomali ${ }^{8}$ para referirse a las casas sagradas timorenses (Castro 1867:317; Lencastre 1931:70). Forbes (1884) traduce el significado de lulik a este concepto emic de origen malayo (Caillois 1984:30; Lombard-Jourdan 1997:100) y denomina "pomali house" (Forbes 1884:411) a las uma-lulik timorenses.

\footnotetext{
6 Lévi-Strauss consideraba que la alianza matrimonial era la relación más importante entre casas. Buena parte de su teoría de la alianza se basaba en la relación entre dadores y tomadores de esposas y la creación de una alianza asimétrica entre ellos, que establecía una jerarquía. Es precisamente en Indonesia Oriental, área cultural en la que tradicionalmente se ha localizado a Timor-Leste, donde encuentra sus ejemplos etnográficos de alianzas matrimoniales (Gillespie 2000:30).

7 El debate sobre las sociedades de casas ha continuado hasta hoy, y muy especialmente en el Sudeste Asiático encontramos múltiples estudios sobre ellas. Para ampliar la información sobre este tema véanse: Ellen (1986), Macdonald (1987), Carsten y Hugh-Jones (1995), Keane (1995) Joyce y Gillespie (2000), Fox (2006b), Vellinga (2004a), Schefold, Domenig y Nas (2004, 2008), Waterson (2009).

8 Es posible que el uso de pomali fuese debido a la utilización del malayo como lengua franca para las actividades comerciales, aunque hoy en día, al menos en mi propia experiencia, se ha perdido totalmente. Esta palabra se encuentra en las obras de misioneros y académicos hasta mediados del siglo XX para traducir la palabra lulik, tanto en portugués (Dores 1907) como en inglés (caso de Forbes) o alemán (Wortelboer 1955).
} 
En la isla, es en Timor Occidental donde primero se lleva a cabo una pionera y muy influyente investigación sobre las casas vernáculas (Aragon y Russell 1999:xli) en tanto que ejes organizadores de la vida social. En el ya clásico Order in the Atoni house, Clark Cunningham (1964) fue uno de los primeros académicos que analizó la casa como sistema simbólico. De igual manera, la casa fue objeto de estudio en varias investigaciones llevadas a cabo en el Timor portugués, de las cuales el libro Arquitectura Timorense (Cinatti, Almeida y Mendes 1987) es uno de los mejores ejemplos. En él se examinan en detalle los principales tipos de casas de las diferentes regiones timorenses. En otro artículo, el propio Cinatti (1965) trató ya el tema de las casas timorenses y los rituales asociados a su construcción; algo de lo que Barros Duarte (1975) se ocupó nuevamente en un estudio de caso en el que describe de forma prolija los elementos arquitectónicos de una casa de la región de Maubisse.

Los antropólogos extranjeros que llegaron al Timor portugués para realizar investigaciones etnográficas (Hicks 2011) se fijaron también en las uma-lulik como institución social y estructura material. En ese sentido, obras como la de Lameiras-Campagnolo (1975), acerca de las técnicas de construcción de las casas entre los fataluku9 de Loré (Lórehe), y la de RenardClamagirand $(1975,1982)$ sobre los kémak de Marobo, ampliaron el conocimiento sobre las casas en distintas regiones y grupos lingüísticos del Timor portugués. Pero es quizás con la llegada de los antropólogos americanos e ingleses cuando se comienza a estudiar las uma-lulik en tanto que instituciones sociales y a analizar su importancia como elemento estructural y de la práctica en las sociedades y culturas timorenses. Así, David Hicks, a lo largo de su amplia obra (Hicks 2004, 2008, 2013), ha reflexionado sobre el papel en la sociedad timorense de las uma-lulik, a las que ha definido como "the ritual house of a descent group, a repository for the group's sacred emblems" (2004:26). ${ }^{10}$ Traube ha visto las fad lisa y fad luli de los mambai de Aileu como la unidad social básica (1980b:294-295, 1989:322-323), y el sistema de casas como un aspecto de su vida ritual (Traube 1986). Forman (1980:154) ha entendido las oma bese como las casas sagradas de un grupo de descendencia (linaje) entre los makassae de Baucau.

Las uma-lulik, hasta la llegada de los portugueses y pese a los contactos de los timorenses con otros pueblos, mantuvieron su preeminencia como elemento central de la organización social. Empero, el colonialismo impuso - no sin dificultades- un nuevo tipo de organización social ajeno al nativo, pasando este último a funcionar de forma subsumida y en ocasiones subrepticiamente bajo el orden colonial. Aunque los timorenses dieron explicaciones cosmológicas a la presencia lusa (Traube 1991), la cultura tradicional y sus instituciones fueron objeto de un intento de asimilación por parte de los portugueses, que produjeron un conocimiento etnológico sobre la cultura tradicional -los usos e costumes- (Roque 2011) que servía a sus propios intereses políticos. En ocasiones, el choque de culturas se materializó en la destrucción de casas sagradas (Delgado Rosa 2012; Fernandes 2014:89-90), y quizás la destrucción del reino de Wehali y con él de sus uma-lulik es el caso más famoso y de más relevancia para el devenir del colonialismo portugués (Castro 1867:34-35).

La ocupación indonesia frenó la investigación extranjera en Timor. No obstante, se continuó realizando estudios, promovidos ahora por el Gobierno indonesio, sobre las casas sagradas,

9 La tesis de licenciatura de Gomes (1972) ya había tratado acerca de la construcción de las casas, a las que denomina "casas artísticas" (1972: 208), entre los fataluku.

10 Más recientemente Hicks (2008:164), influenciado por la obra de Vellinga (2004a), ha explorado la manera en que las casas sagradas funcionan como un agente activo y no meramente un receptor pasivo en la constitución identitaria de grupos sociales e individuos. 
que fueron objeto de interés en varias publicaciones, tales como Susilo (1991) o Susilio et al. (1997). Especialmente durante los años noventa, se llevó a cabo varios proyectos de investigación organizados desde el Ministerio de Educación y Cultura indonesio para inventariar y poner en valor diversos elementos de la sociedad tradicional timorense. Muchas de estas investigaciones estetizaban e incluso comodificaban prácticas, conocimiento local e instituciones sociales timorenses para que pudiesen servir a los intereses del Estado indonesio. ${ }^{11}$ Es conocido en particular el uso con fines propagandísticos que Indonesia realizó de la tradición e historia timorenses para legitimar su ocupación (Gunter 2007; McWilliam 2005).

\section{El estado y la CULTURA tRADICIONAL EN INDONESIA COMO PUNTO DE REFERENCIA}

En su artículo "Culture as Art: From Practice to Spectacle in Indonesia" (1985), Greg Acciaioli escribía acerca del relativo declinar de la autoridad basada en el adat durante los años ochenta en una región central de Célebes, en comparación con el estatus de dicha autoridad durante el período colonial holandés. Argumentaba que las políticas de la Indonesia de Suharto en relación con el adat tenían como intención situarlo dentro del plano de lo artístico o lo patrimonial, estableciendo así un marco limitado y controlado en el que estas se podían desarrollar sin, de ese modo, poner en cuestión los intereses del Estado. ${ }^{12}$ Mediante la estetización de la cultura tradicional, decía Acciaioli, se producía un vaciado de contenido en el adat, que quedaba relegado a una expresión meramente artística o patrimonial: "culture has become art throughout Indonesia, but for the most peripheral even that art has been prescribed by the state" (Acciaioli 1985:162).

Aunque encontró eco en algunos trabajos de otros autores como Spyer (1996) y Graham (1994), el planteamiento de Acciaioli también fue contestado por Yampolsky (1995) y Aragon (1996). Yampolsky (1995:714) sostuvo que le parecía poco creíble que la intención de la política del Gobierno indonesio en relación con las sociedades tradicionales fuese la de "castrar" el adat y que, por tanto, Acciaioli exageraba acerca de este asunto. Si bien se mostraba de acuerdo con que las políticas del Gobierno indonesio podrían tener ese efecto en el futuro, discrepaba en lo relativo a que la estetización del adat lo vaciase de contenido de forma que perdiese sus funciones prácticas en ese proceso. Aragon, por su parte, centrándose más en el choque entre los sistemas de creencias tradicionales y la religión protestante entre los tobaku-vecinos de los to-lindu estudiados por Acciaioli-, proponía que a pesar de que los rituales cristianos habían sustituido en buena medida a los tradicionales, era la lógica indígena la que dictaba su contexto de representación..$^{13}$ En el caso de los tobaku, dice (1996:435): "these revivals [de representaciones estetizadas, en este caso en forma de canciones tradicionales] are stolen opportunities for locals

11 Véanse, entre otros, da Crus y Susilo (1991) sobre la artesanía; Hidayah et al. (1990) sobre las ceremonias; y Gusman et al. (1991) sobre la medicina tradicional.

12 Intereses en muchas ocasiones espurios, como muestran los amplios casos de abuso de poder del Estado indonesio contra las sociedades indígenas para introducir proyectos mineros o madereros en sus tierras.

13 Más recientemente, el propio Acciaioli (2002) ha revisado su postura en relación con el artículo que cito y sostenido que la visión que tenía en 1985 no era incorrecta en el contexto de las políticas culturas de la Indonesia del Nuevo Orden durante los años ochenta. Registra, asimismo, un resurgir del adat entre los to-lindu durante la década de los noventa que sitúa en la utilización del adat como una estrategia de empoderamiento de las comunidades frente al Estado, una suerte de "arma de los débiles" (Scott 1985) contra las políticas estatales abusivas. E1 adat ha servido como legitimación -ayudado por la sociedad civil- para reivindicar los derechos sobre la tierra de las comunidades to-lindu frente al ordenamiento jurídico del Estado. 
to circulate their knowledge of precolonial cosmology, as well as define their ethnic identities".

Esta estetización de la cultura tiene uno de sus ejemplos más conocidos en la arquitectura vernácula indonesia, que durante el Nuevo Orden fue promocionada efusivamente. La celebración de la diversidad de dicha arquitectura fue un importante elemento retórico (Adams 1998) para conmemorar la unidad nacional de Indonesia bajo el motto nacional (Acciaioli 1985:227) Bhinneka Tunggal Ika ('Unidad en la diversidad'). En el festejo de esta diversidad arquitectónica, sostiene Keane (1995), el Estado encontró ejemplos testimoniales de una "diferencia étnica inocua”. Un claro ejemplo de esta celebración es el Parque de la hermosa Indonesia en miniatura (Tama Mini Indonesia Indab), un parque de atracciones de base cultural en Yakarta en el que se presenta una selección de reconstrucciones de las distintas casas tradicionales (rumah adat) del archipiélago a una escala enorme. El parque, han señalado Davidson \& Henley (2007:10), "had an abstract, symbolic quality, reducing local cultures to a standardized spectacle of picturesque 'adat houses' and 'adat costumes' En 1980 se abrió en este parque la sección de Timor Oriental, que estaba representada por la casa tradicional de Lospalos y que aún está presente hoy en día (TMII 2012). La presencia de la casa timorense en el parque sirvió, en palabras de McWilliam, para "contain and integrate Timorese difference within an authorised framework of cultural performance” (McWilliam 2005:31).

Estas políticas en relación con las culturas tradicionales del Nuevo Orden indonesio tenían mucho que ver con una visión turística de la cultura (Erb 2007:252), lo cual tiene su ejemplo más claro en la cultura balinesa. Picard (1997) ha argumentado que la formación de la identidad balinesa no puede entenderse dejando a un lado su aspecto turístico, y llega a sostener que la propia cultura balinesa es un "artefacto cultural" (1997:185). Esta visión turística de la cultura también puede verse en el caso de Timor, donde el Gobierno indonesio llegó a promover la realización un informe (Windiyarti et al. 1993) sobre el impacto del turismo en la sociedad tradicional timorense.

\section{La Academia Nacional de Arte e Industrias Creativas. La traslación de la COMPETICIÓN DE ESTATUS DEL NIVEL LOCAL AL NACIONAL}

En un artículo reciente (Alonso Población y Fidalgo Castro 2014), hemos revisado cómo el capital narrativo en relación con las narrativas de origen juega un papel de suma importancia para entender las dinámicas de los sistemas de precedencia (Acciaioli 2009; Fox 1994) en una comunidad de Liquiçá. En este sentido, considero que el siguiente caso presenta un ejemplo tangible de cómo el capital narrativo detentando por individuos particulares en tanto que miembros de un grupo de descendencia supone un desafío para dotar de valor patrimonial algunos elementos culturales, especialmente las uma-lulik y las narrativas orales:

En julio de 2011, mientras me encontraba realizando la investigación de campo para mi tesis doctoral, fui invitado a moderar un debate sobre arquitectura tradicional que se celebró en la I Conferencia sobre la Academia Nacional de Arte e Industrias Creativas. En él participaban un lia-na'in ${ }^{14}$ de una uma-lulik

14 Los lia-na'in son uno de los diferentes tipos de autoridades tradicionales que existen en Timor Oriental. El término ha sido traducido en muchas ocasiones como "señor de la palabra". Creo, sin embargo, que serían más correctas otras traducciones tales como "el que sabe hablar", "el que puede hablar", "guardián/custodio de la palabra", "el que tiene derecho de hablar", "orador". En un trabajo anterior (Fidalgo Castro 2012) trato más ampliamente 
1lamada Dato Muras Haimolin de Fohorem, dos representantes de la Secretaría de Estado de la Cultura y un carpintero tradicional. El objetivo del debate era presentar los aspectos patrimoniales que representan las uma-lulik de Timor en sus dos vertientes, la material y la intangible, para conseguir con ello una puesta en valor de la arquitectura tradicional y el conocimiento local asociado a ella. Para esto, cada uno de los miembros de la mesa elaboró una breve presentación de aspectos relacionados con las uma-lulik.

Una vez terminada la presentación, en la que el lia-na’in recitó una narrativa de origen asociada a su casa, se abrió el turno para la ronda de preguntas. Uno de los intervinientes me hizo de manera airada, después de incorporarse para hablar, la siguiente pregunta: Despois Ita-boot nia peskiza kona-ba uma-lulik remata ona, tuir Ita-boot nia haree... uma-lulik iha Timor ne'e... Ida néebé mak boot liu? ('Después de haber terminado su investigación, según su punto de vista... de entre todas las uma-lulik de Timor... ¡cuál es la más importante?'). Un segundo interviniente criticó el método de selección por el que se había decidido que fuese el lia-na’in de la uma-lulik Dato Muras Haimolin presente en la conferencia y no otro el que recitase su narrativa de origen, ya que su casa no era "la más importante" de Timor (pertenecía a una casa de Fohorem, en el distrito de Cova Lima), y pidió que se explicase cuáles habían sido los criterios utilizados para elegirlo. La intervención de nuevas personas, todas en esta misma línea, me llevó a explicar que la selección no se había debido a ninguna causa concreta, sino que la casa había sido elegida al azar con el objetivo de dar a entender la riqueza de las istória sira ('narrativas de origen') a los extranjeros que allí se encontraban, para que tuviesen un ejemplo de la riqueza de la kultura Timor nian ("cultura de Timor"). Solo después de esta explicación, que fue más larga y razonada, comenzó la gente a calmarse.

La selección en este caso de la casa mencionada como ejemplo para poner en valor la literatura oral timorense -sus mitos y narrativas- generó claras tensiones entre los asistentes. Las personas intervinientes eran conscientes de que la casa Dato Muras Haimolin, al haber sido escogida para presentar su narrativa de origen en un evento de nivel nacional, potenciaba con ello su capital simbólico, por eso incidían en cuestionar su importancia en relación con el capital narrativo que detentaba, que era puesto en duda y minusvalorado implícitamente mediante sus discursos. Nos encontramos, por tanto, que se había producido en el nivel nacional una competición de estatus entre casas (Vellinga 2004b), algo que normalmente se dirime en un nivel regional o local.

\section{La reconstrucción de la uma-lulik Nunufú en Hatubuilico}

En un artículo reciente (2013), David Hicks lamenta la falta de hibridación entre las culturas políticas del adat y del Estado-Nación. Esta mezcla, sostiene, podría dar lugar a una nación híbrida fruto del sincretismo. A pesar de mostrarse pesimista por el poco interés del Gobierno y de las Naciones Unidas a la hora de introducir elementos del adat dentro de los procesos de gobernabilidad y justicia, muestra cierto optimismo ante una nota de prensa de la Embajada de Estados Unidos en Dili (2013: 24) en que puede leerse lo que sigue:

Sacred House Inaugurated in Hatobuilico, April 23, 2010

The Embassy and the Culture Secretariat collaborated to refurbish the uma-lulik as part of a joint effort to preserve important elements of Timor-Leste's traditional culture. Uma-luliks in Oecussi, Bobonaro and Lautem are part of the same cultural preservation project. The total cost is $\$ 32,000$.

Uma-luliks are the spiritual center of communities throughout Timor-Leste. Each village uses its uma-lulik as a gathering place for traditional rituals and as a spiritual resting place for the village ancestors. The United States provides financial support for uma-lulik preservation out of respect for the roots of Timorese national and cultural identity. (Embassy of the United States in Dili 2010)

acerca de este concepto, en tanto que parte de los compuestos del sufijo -na’in. 
Esta nota de prensa que Hicks refiere venía precedida por otro anuncio del Gobierno de Timor-Leste del día anterior (22 de abril), en que se mencionaba que la casa de Bobonaro, que el embajador había visitado junto con el secretario de Estado de la Cultura, se denominaba Lokometa-Darlau Nunufú (Timor-Leste 2010). Esta casa era una de las cuatro uma-lulik que habían sido beneficiarias de una ayuda total de 32000 dólares para apoyar su reconstrucción, donada por la Embajada de Estados Unidos en Dili con cargo al U.S. Ambassadors Fund for Cultural Preservation de 2008 (Embassy of the United States in Dili 2009) y gestionada por la Secretaría de Estado de la Cultura. Mientras se producía la reconstrucción de dicha casa sagrada, yo me encontraba realizando el trabajo de campo de un proyecto de cooperación financiado, entre otras, por la Agencia Española de Cooperación Internacional al Desarrollo (AECID) en colaboración con la Secretaría de Estado de la Cultura. ${ }^{15} \mathrm{El}$ distrito de Ainaro fue uno de los lugares donde se efectuó el trabajo de campo y el conjunto de casas mencionado en la nota de prensa fue uno de los que visitamos en esta investigación.

Junto con otros miembros del equipo, me había desplazado a realizar una visita y entrevistas de campo a un clúster de casas (uma-knua) llamado Darlau-Lebolau, junto con sus uma-na'in ('dueños de la casa'). En él se encontraban tres casas denominadas Loko-Meta, Darlau y Nunufú que eran tres unidades diferenciadas, y no como señalaba el anuncio del Gobierno una sola, y que mantenían entre ellas una relación asimétrica establecida mediante una narrativa de origen que creaba un orden de precedencia. Esta precedencia se expresaba mediante la utilización de una metáfora de familia, según la cual Loko-Meta y Darlau eran las casas "madre" (uma ferik) y "padre" (uma katuas) respectivamente, mientras que Nunufú era la "casa hija” (uma oan) (Pena Castro 2010b:123). En esta relación de precedencia, Darlau era considerada la más importante, seguida de Loko-Meta y finalmente Nunufú.

El lugar no estaba habitado debido a lo remoto de su localización, y los miembros de las casas se habían asentado en lugares más cercanos a la villa de Hatubuilico. En el lugar originario solo quedaba en pie -aunque en mal estado-Darlau. De las otras dos apenas permanecían restos derruidos por el paso del tiempo. Loko-Meta había sido reconstruida (Gárate Castro 2010:105), antes de la independencia, en otro paraje más cercano a la villa, después de realizar una ceremonia para solicitar permiso a los antepasados (beíala) para trasladarla de lugar. Cuando preguntamos si Darlau y Loko-Meta habían sido también reconstruidas en otro sitio, varios de los lia-na'in de Darlau que estábamos entrevistando mostraron su enfado ante el hecho de que Nunufú estuviese siendo reconstruida con apoyo del Gobierno. Al inquirir a qué se debía el enfado, uno de ellos nos explicó que comenzar a reconstruir las casas por la última en el orden de precedencia era incorrecto (sala); es decir: al subvertir el orden de precedencia se desequilibraba también el orden cosmológico y social. Lo correcto, decía, habría sido empezar por Darlau, continuar por Loko-Meta y finalizar por Nunufú.

No nos interesa para este caso establecer los motivos que llevaron a que las tres casas no decidiesen colegiadamente cuál de ellas debía haber sido la primera en reconstruirse. Las causas pueden ser múltiples: conflictos políticos, conflictos familiares o incluso la pugna por acceder a la propia ayuda gubernamental para la reconstrucción. Para el caso, lo que nos interesa señalar es el hecho de que la intervención del Gobierno abrió (o reabrió) un conflicto al entregarle la ayuda económica a la última de las casas del clúster en el orden de precedencia, pues algunos

15 Ese proyecto incluía el levantamiento de datos y la publicación de dos libros acerca de las uma-lulik de dos distritos de Timor. Finalmente solo vio la luz uno de los dos libros previstos, Património cultural de Timor-Leste. As uma lulik do distrito de Ainaro (Gárate Castro y Assís 2010). 
miembros de las otras casas vieron dicha entrega como el otorgamiento a esta casa de un marcador de estatus nuevo, ajeno al marcador de la narrativa de origen (sagrada) que define el orden de precedencia de las casas. Por eso, la ayuda del Gobierno se ve como un ataque a la legitimidad basada en la narrativa de origen, como un intento de contestación a la jerarquía tradicional mediante la introducción de elementos ajenos a ella.

Por otra parte, el proceso de construcción de una uma-lulik va acompañado de un largo proceso de rituales que, como Traube ha señalado (1986), son un elemento esencial de la socialidad timorense. Es a través de ellos y de las relaciones entre casas (fetosaa-umane, maun-alin $)^{16}$ que se movilizan los recursos para la construcción de nuevas casas. Traube (1980a:101-102, 1986:204-205) ha señalado que uno de los objetivos de un grupo de descendencia a la hora de llevar a cabo un ritual es el de obtener ganancias económicas. Mediante la movilización de las redes de parentesco presentes en el sistema de casas, los organizadores de un ritual redistribuyen los recursos económicos entre los dadores y tomadores de esposas de tal modo que, al dar por finalizada la ceremonia, se produce una ganancia económica. La introducción de ayudas ajenas a esta red de intercambios pone en cuestión la necesidad de movilizar el complejo sistema de casas para reconstruir una uma-lulik, y, de ese modo, contesta la necesidad de mantener las relaciones entre casas.

Mediante este pequeño ejemplo he pretendido mostrar cómo el orden de precedencia y jerarquía que se establece y reproduce mediante el adat no está fuera de contestación y es subvertido voluntaria y conscientemente por algunos individuos en función de sus intereses (en este caso hacerse con una ayuda económica de 8000 dólares americanos). Este es un caso que ilustra cómo, en palabras de García Canclini, "el patrimonio cultural funciona como recurso para reproducir diferencias entre los diferentes grupos sociales y la hegemonía de quienes logran un acceso preferente a la producción y distribución de los bienes" (2001:187).

El apoyo a la reconstrucción de la uma-lulik de Hatubuilico por parte de la Embajada de Estados Unidos en Dili tenía como intención promocionar la cultura tradicional mediante una intervención patrimonial. Sin embargo, esta intervención sirvió para contestar el orden de precedencia que la propia cultura tradicional establecía, generando con ello una situación de conflicto. La política nacional de la cultura menciona que "quanto melhor for a compreensão dos vários elementos culturais [...] menor será a possibilidade de existirem tensões sociais, contribuindo-se deste modo para o objetivo de construir um Estado verdadeiramente multicultural, desenvolvido e pacífico" (RDTL 2009). El conocimiento acerca de las casas sagradas es complejo y requiere un conocimiento profundo de la vida social timorense, además de otros elementos histórico-políticos que hay que tener en cuenta. Es por esto que las intervenciones patrimoniales deben ser, como ha señalado Pena Castro,

un ejercicio compartido de negociación social, que se acentúa en tanto que hablamos de elementos que todavía juegan un papel destacado en la articulación contemporánea de las identidades locales. No se trata de oscuros vestigios del pasado, sino de bienes contemporáneos activos en la ordenación social vigente. (Pena Castro2010a:330)

García Canclini (2001:186-194) ha propuesto que, a la luz de las teorías de la reproducción cultural y la desigualdad social -especialmente Bourdieu-, el patrimonio reformulado en

16 En la tesis doctoral de Lúcio Sousa (2010) encontramos una amplia exploración etnográfica de la relevancia da casa en los niveles social, simbólico, identitario y práctico. 
tanto que capital cultural puede ser entendido como proceso social. Frente a una idea de patrimonio estática, que no tiene en cuenta las transformaciones ocurridas en las comunidades locales (Sousa 2007:225), su comprensión como capital cultural supone abrirlo al análisis de los usos sociales de los cuales es objeto: cómo se transforma, cómo los distintos grupos sociales se apropian de él de manera desigual. Entenderlo, en fin, como un elemento de poder.

\section{El informe Roads for Cultural Heritage: Policy Note on Cultural Heritage and Infrastructure Development in Timor-Leste del Banco Mundial}

Según el informe del Banco Mundial Roads for Cultural Heritage: Policy Note on Cultural Heritage and Infrastructure Development in Timor-Leste (2013), su objetivo es este: "survey Timor-Leste's cultural heritage in relation to infrastructure development and offer policy options for conserving and developing cultural heritage assets, particularly in relation to sustainable tourism" (World Bank 2013:3). Uno de los elementos que destaca como especialmente interesante para conseguir este objetivo es el de las casas sagradas, y refiere un pequeño ejemplo de una uma-lulik que podría servir como modelo para el desarrollo de proyectos de turismo cultural como estrategia de conservación del patrimonio. Selecciono solo algunas partes del texto:

Annex 2 Example of High Potential Heritage Asset - Lacoto Sacred House

Lacoto is a sacred house (uma-lulik) located on the outskirts of Dili district. The Lacoto clan histories speak of their ancestry as the original inhabitants of Dili [...]. During the Indonesian occupation period the sacred house was used to protect Kay Rala Xanana Gusmao and other resistance leaders as they were conducting military activities close to Dili. In this way, the Lacoto sacred house represents both the key role of customary culture during the resistance period, and post-independence revival of tradition. These factors, as well as the location convenient to Dili, suggest that it has good potential to attract tourists. As the site is under customary ownership by the Lacoto clan, and clan leaders (such as hereditary seniors and ritual specialists) have responsibility for ensuring the appropriate use of the site, the assessment recommends that these figures, with facilitation from SSAC, be mobilized to lead the development of a community organization and management plan for the site. [...]

The development of uma-lulik Lacoto for sustainable tourism can contribute to improving international understanding of Timor-Leste's land and people, and an important part of this process is to establish a tourism experience that is both sensitive to local custom and sensitive to the aspirations of tourists. (World Bank 2013:11)

El papel del turismo cultural como estrategia de empoderamiento ha sido estudiado por Adams (2006) entre los toraya de Indonesia. Esta autora critica la concepción de los toraya como receptores pasivos de políticas culturales y turísticas impuestas sobre ellos, y demuestra que, muy lejos de esa pasividad, han sabido aprovechar el turismo políticamente, haciendo de él un mecanismo y un canal de comunicación que les permite inscribirse dentro de la historia y las políticas indonesias. Aunque es cierto que el turismo cultural y comunitario propuesto en el informe del Banco Mundial podría tener algunos efectos empoderadores para las comunidades locales, no lo es menos que los actores externos a la comunidad -intermediarios, agentes culturales o empresarios turísticos- pueden ejercer su poder iniciando procesos de comodificación que pueden llevar a la explotación de las comunidades locales y sus miembros (Wilson y Ypeij 2012). 
Existe, por otra parte, el problema del probado efecto etnogenerador ${ }^{17}$ que el turismo tiene sobre las comunidades locales (Adams 1997; Erb 1998; Picard 1997). Hay ejemplos cercanos acerca de las consecuencias que este tipo de procesos pueden traer consigo en determinadas circunstancias, como es el caso de la violencia interétnica entre las comunidades indígenas dayak de Kalimantan (Borneo indonesio) contra los transmigrantes ${ }^{18}$ madureses de Java Occidental (Henley y Davidson 2007b). Si bien no se puede hablar de etnias en Timor Oriental, una posible potenciación de las identidades locales podría generar serios problemas de seguridad y marginación entre grupos. En Timor Oriental la crisis de 2006 puede ser leída, siquiera parcialmente, en clave de conflicto protoétnico bajo las identidades loromonu y lorosa'e. ${ }^{19}$ En ese sentido, no puedo sino estar de acuerdo con Bovensiepen cuando afirma que "recent initiatives to build a national lulik house and to collect data on indigenous culture may well lead to a stronger identification and perhaps commoditization of local communities as divided into 'ethnic groups' with shared origins and traditions" (2014:302).

\section{Conclusión}

En este artículo he pretendido mostrar la dificultad de articular dos tipos de sistemas sociales diferentes: el representado por el Estado-Nación timorense y el representado por el adat. Para esto he utilizado uno de los elementos centrales de la organización social tradicional, la uma-lulik, cuyo concepto he intentado delimitar. En ese proceso he señalado cómo la cultura timorense ha sido tratada por parte de los poderes coloniales (Portugal e Indonesia) como un elemento estetizado, con la intención de subsumirla dentro de su propio orden social, adscribiéndola a una esfera controlada de expresión. Mediante la revisión del proceso de estetización de la cultura entre los toraya de las islas Célebes he pretendido, asimismo, establecer paralelismos con las políticas culturales que se están llevando a cabo en Timor Oriental. La clasificación de la cultura tradicional dentro de las esferas del arte y el patrimonio, y su asociación al turismo, que la política cultural timorense parece realizar, son, en buena medida, recetas repetidas de las políticas llevadas a cabo durante el período del Nuevo Orden indonesio.

Posteriormente he utilizado algunos estudios de caso para llamar la atención sobre la resistencia de los agentes de la cultura timorense a ser reducidos a elementos patrimoniales o artísticos. En el primero, muestro cómo el intento de poner en valor las narrativas orales choca con los usos políticos de legitimación que los timorenses hacen de ellas. Las narrativas de origen, asociadas a casas sagradas particulares, han sido clasificadas en la política nacional de la cultura como parte del patrimonio inmaterial timorense; no obstante, la "propiedad" de esas narrativas es algo que aún está por dirimirse. En el contexto regional de Timor-Leste encontramos un

17 Sobre el concepto y proceso de etnogénesis, véanse Roosens (1989) y Peel (1989).

18 Durante la década de los setenta y ochenta, Indonesia lanzó los programas de transmigración (tansmigrasi), que consistían en el reasentamiento de población de unas islas en otras. Los objetivos explícitos de los programas de transmigración, patrocinados por el Banco Mundial, eran el fomento del desarrollo agrícola y el alivio de la presión demográfica en islas superpobladas como Bali y Java (Henley y Davidson 2007a, 2007b; Levang 1997).

19 Sobre este asunto, menciona Prüller (2008:78) lo siguiente: "This paper has shown that deeply-felt ethnic hatred is not at the heart of the problem. Nevertheless, the categories in discussion have been found to have precedented the 2006 crisis by many years. Firaku and Kaladi are not merely fantasies in current political rhetoric, as Hicks (2007) argues, but identity concepts that have been loaded with highly explosive content in post-independent East Timor". 
ejemplo similar en el caso de la aprobación de la Ley sobre los derechos de autor de Indonesia ${ }^{20}$, cuyas implicaciones han sido analizadas por Lorraine V. Aragon (2012). Esta ley se apropia para el Estado de los derechos de autor del folclore y los productos culturales populares, basándose en la protección del patrimonio nacional. Sin embargo, artistas regionales y artesanos han contestado fuertemente esta visión, ya que no consideran que sus conocimientos o prácticas sean susceptibles de ser objeto de apropiación exclusiva por parte de individuos o grupos, incluido el propio Estado.

En el segundo busco poner de manifiesto cómo una intervención cuyo objetivo es la protección del patrimonio puede, paradójicamente, romper todos los elementos sociales que han sostenido dicho patrimonio hasta la fecha y generar tensiones sociales al propiciar la subversión del orden social de precedencia. Finalmente muestro cómo las políticas de protección patrimonial asociadas al turismo cultural podrían generar problemas sociales en dos niveles: el de la explotación de los beneficiarios últimos y el de creación de identidades étnicas. Este último caso es quizás el más relevante de cara a la política cultural de la República Democrática de Timor-Leste, ya que la preservación patrimonial tiene como objetivo explicitado la "defesa e consolidação da unidade e identidade Nacionais". La ausencia de identidades locales (indígenas) en el aparato legal del Estado timorense ha llevado a que estas hayan quedado subsumidas dentro de la categoría de "ciudadanía" (Silva y Simião 2012). La estetización de las culturas locales timorenses podría tener, al igual que en el caso de la toraya, el inesperado efecto de generar diversas identidades étnicas donde se quiere potenciar la existencia de una identidad común, no excluyente: la timorense.

Todos los casos señalados apuntan en la misma dirección: los problemas de llevar a la práctica acciones fundamentadas sobre la consideración de las uma-lulik como patrimonio nacional timorense. Antonio A. Arantes ha señalado en diversos lugares (Arantes 2007, 2008, 2013) muchas de las dificultades que la tarea de la patrimonialización, especialmente de la cultura intangible, comporta. Analiza Arantes, hablando acerca de la legislación brasileña sobre el patrimonio (2007:293), la complejidad cultural y política de poner en marcha prácticas de protección del patrimonio, debido a que se trata de elementos vivos de las sociedades en la se encuentra: "heritage [ ] belongs to communities and its embedded in the social and cultural processes by which their social lives are produced and transdormed". Las intervenciones de política patrimonial requieren de una profunda negociación y mediación cultural entre todos los actores implicados para crear, a través de la experiencia del patrimonio cultural, una comunidad de la memoria (Lowenthal 1998) más plural e inclusiva, en definitiva, más compleja.

\section{Bibliografía}

Acciaioli, Greg. 1985. "Culture as Art: From Practice to Spectacle in Indonesia." Canberra Anthropology 8(1 \& 2):148-72.

Acciaioli, Greg. 2002. "Re-Empowering the 'Art of the Elders': The Revitalisation of Adat among the To Lindu People of Central Sulawesi and Contemporary Indonesia." Pp. 217-44 en Beyond Jakarta: Regional Autonomy and Local Society in Indonesia, editado por Minako Sakai. Adelaide: Crawford House.

20 Undang Undang Republik Indonesia Nomor 19 Tabun 2002 tentang Hak Cipta. 
Acciaioli, Greg. 2009. "Distinguishing Hierarchy and Precedence: Comparing Status Distinctions in South Asia and the Austronesian World, with Special Reference to South Sulawesi." Pp. 51-90 en Precedence. Social Differentiation in the Austronesian World, editado por Michael P Vischer. Canberra: ANU E Press. Consulta: (http://epress.anu.edu.au/ precedence_citation.html).

Adams, Kathleen Marie. 1997. “Touting Touristic 'Primadonas': Tourism, Ethnicity, and National Integration in Sulawesi, Indonesia." Pp. 155-80 en Tourism, Ethnicity and the State in Southeast Asia and the Pacific, editado por M. Picard y R. Wood. Honolulu: University of Hawai'i Press.

Adams, Kathleen Marie. 1998. "More than an Ethnic Marker: Toraja Art as Identity Negotiator.” American Ethnologist 25(3):327-51.

Adams, Kathleen Marie. 2006. Art as Politics. Re-Crafting Identities, Tourism, and Power in Tana Toraja, Indonesia. Honolulu: University of Hawai'i Press.

Alonso Población, Enrique y Alberto Fidalgo Castro. 2014. "Webs of Legitimacy and Discredit: Narrative Capital and Politics of Ritual in a Timor-Leste Community." Anthropological Forum 24(3):245-66. Última consulta: 22 de agosto de 2014. (http://www.tandfonline. com/doi/abs/10.1080/00664677.2014.948381).

Do Amaral de Carvalho, Demetrio y Jose Coreia. 2011. “Tara Bandu Nudar Matenek Ekologia Tradisional (Lokal).” Pp. 52-67 en Matenek Lokal Timor nian! Haktuir husi (Links) workshop "Matenek Tradisional Riku soin ba Dezenvolvimentu" Dili, 7-8 Junhu 2011, editado por Demetrio do Amaral de Carvalho. Jakarta: Haburas Foundation, Timor Leste National Commission for UNESCO and the UNESCO Regional Science Bureau for Asia and the Pacific in Jakarta.

Anon. 2012. "President Vetoes Three Land Laws. Prezidente Veto Lei Rai Tolu." La'o Hamutuk. Última consulta: 1 de enero de 2015. (http://www.laohamutuk.org/Agri/ land/2012/12PNpassPRveto.htm).

Aragon, Lorraine Victoria. 1996. "Suppressed and Revised Performances: Raego' Songs of Central Sulawesi.” Ethnomusicology 40(3):413-39. Consulta: (http://www.jstor.org/stable/852470).

Aragon, Lorraine Victoria. 2012. "Copyrighting Culture for the Nation? Intangible Property Nationalism and the Regional Arts of Indonesia." International Journal of Cultural Property 19(3):269-312. Última consulta: 1 de septiembre de 2014. (http://www.journals.cambridge.org/abstract_S0940739112000203).

Aragon, Lorraine Victoria y Susan D. Russell. 1999. "Introduction. Delineating Theoretical Approaches to Southeast Asian Anthropology." Pp. xxiii - lxii en Structuralism's Transformations. Order and Revision in Indonesian and Malaysian Societies. Papers Written in Honor of Clark E. Cunningham, editado por Lorraine Victoria Aragon y Susan D. Russell. Arizona State University. Program for Southeast Asian Studies, Monograph Series Press. Arantes, Antonio A. 2007. "Diversity, Heritage and Cultural Politics." Theory, Culture E Society 24(7-8):290-96. Última consulta: 2 de enero de 2015. (http://tcs.sagepub.com/cgi/doi/10 $.1177 / 02632764070240072506)$.

Arantes, Antonio A. 2008. "African-Brazilian Cultural References In National Heritage Questions of Cultural Politics." Vibrant 5(1):20-33. Consulta:(http://www.vibrant.org. br/issues/v5n1/antonio-a-arantes-african-brazilian-cultural-references-in-national-heritage/).

Arantes, Antonio A. 2013. "Beyond Tradition: Cultural Mediation in the Safeguarding of ICH.” Pp. 39-55 en Anthropological Perspectives on Intangible Cultural Heritage, vol. 6, SpringerBriefs in Environment, Security, Development and Peace, editado por Lourdes 
Arizpe y Cristina Amescua. Heidelberg: Springer International Publishing. Última consulta: 2 de enero de 2015. (http://link.springer.com/10.1007/978-3-319-00855-4).

Babo-Soares, Dionísio da Costa. 2004. "Nahe Biti: The Philosophy and Process of Grassroots Reconciliation (and Justice) in East Timor." The Asia Pacific Journal of Anthropology 5(1):15-33.

Babo-Soares, Dionísio da Costa. 2007. "O desenvolvimento do setor de justiça no Timor Leste." Pp. 193-209 en Timor-Leste por trás do palco: cooperação internacional e a dialética da formação do estado, editado por Kelly Cristiane da Silva y Daniel Schroeter Simião. Belo Horizonte: Editora UFMG.

Barros Duarte, Jorge. 1975. "Casa Turi-Sai - Um tipo de casa timorense.” Garcia de Orta. Série de Antropologia 2(1-2):1-33.

Bovensiepen, Judith M. 2014. "Installing the Insider 'outside': House Reconstruction and the Transformation of Binary Ideologies in Independent Timor-Leste." American Ethnologist 41(2):290-304. Última consulta: 22 de julio de 2014. (http://doi.wiley.com/10.1111/ amet.12076).

Caillois, Roger. 1984. El hombre y lo sagrado. México: Fondo de Cultura Económica.

Carsten, Janet y Stephen Hugh-Jones. 1995. About the House. Lévi-Strauss and beyond. Cambridge: Cambridge University Press.

Castro, Affonso de. 1867. As possessões portuguezas na Oceania. Imprensa Nacional.

Cinatti, Ruy Vaz Monteiro Gomes. 1965. "Tipos de casas timorenses e um rito de consagraçao.” Pp. 155-79 en Actas do Congresso Internacional de Etnografia Promovido pela Câmara Municipal de Santo Tirso de 10 a 18 de Julho de 1963. Vol. IV: Colóquio de Etnografia Comparada, vol. IV. Lisboa: Junta de Investigações do Ultramar.

Cinatti, Ruy Vaz Monteiro Gomes, Leopoldo de Almeida, y Sousa Mendes. 1987. Arquitectura timorense. Lisboa: Instituto de Investigacao Cientifica Tropical, Museu de Etnologia.

Cribb, Robert y Audrey Kahin. 2004. Historical Dictionary of Indonesia. $2^{\mathrm{a}}$ ed. Maryland, Toronto \& Oxford: The Scarecrow Press, Inc.

Da Crus, Mario Lopes y Paulus Susilo. 1991. Pengrajin Tradisional Daerab Timor Timur.Jakarta: Departemen Pendidikan dan Kebudayaan, Direktorat Jenderal Kebudayaan, Direktorat Sejarah dan Nilai Tradisional, Proyek Inventarisasi dan Dokumentasi Kebudayaan Daerah.

Cunningham, Clark E. 1964. "Order in the Atoni House." Bijdragen tot de Taal-, Land- en Volkenkunde 120(1):34-68.

D’Andrea, Claudia, Oscar da Silva, y Laura Suzanne Meitzner Yoder. 2003. “The Customary Use of Natural Resources in Timor Leste." en "Land Policy Administration for pro-Poor Rural Growth," Dili, December 2003.

Davidson, Jamie S. y David Henley. 2007. The Revival of Tradition in Indonesian Politics. The Deployment of Adat from Colonialism to Indigenism. Oxon: Routledge.

Delgado Rosa, Frederico. 2012. "Uruvatju e tjiapu: Genealogias invisíveis da etnografia missionária em Timor-Leste.” Pp. 11-39 en Léxico Fataluco-Português, editado por Alberto Fidalgo Castro y Efrén Legaspi Bouza. Dili: Salesianos de Dom Bosco Timor-Leste.

Dores, Raphael das. 1907. Diccionario Teto-Português. Lisboa: Imprensa Nacional.

Ellen, Roy Frank. 1986. "Microcosm, Macrocosm and the Nuaulu House: Concerning the Reductionist Fallacy as Applied to Metaphorical Levels." Bijdragen tot de Taal-, Land-en Volkenkunde 142(1):1-30.

Embassy of the United States in Dili. 2009. "Ambassador Promotes Cultural Preservation in Timor-Leste." Última consulta: 5 de septiembre de 2014. (http://timor-leste.usembassy. gov/news-events/press-releases-2009/ambassador-promotes-cultural-preservation-intimor-leste-february-13-2009.html). 
Embassy of the United States in Dili.2010. “Sacred House Inaugurated in Hatobuilico.”Última consulta: 5 de septiembre de 2014. (http://timor-leste.usembassy.gov/news-events/pressreleases-2010/sacred-house-inaugurated-in-hatobuilico-april-23-2010.html).

Erb, Maribeth. 1998. "Tourism Space in Manggarai, Western Flores, Indonesia: The House as a Contested Place.” Singapore Journal of Tropical Geography 19(2):177-92. Consulta: (http:// www.blackwell-synergy.com/links/doi/10.1111/1467-9493.00033).

Erb, Maribeth. 2007. "Adat Revivalism in Western Flores. Culture, Religion, and Land." Pp. 247-74 en The Revival of Tradition in Indonesian Politics. The deployment of adat from colonialism to indigenism, editado por Jamie S. Davidson y David Henley. Oxon: Routledge.

Fernandes, Alexandre Jorge de Medeiros. 2014. "Em searas do Timor Português: Um estudo sobre as práticas de mediação da Diocese de Díli no período colonial (1949-1973).” MA dissertation, Instituto de Ciências Sociais, Departamento de Antropologia, Programa de Pós-Graduação em Antropologia Social, Universidade de Brasília. Última consulta: 6 de semtiembre de 2014. (http://hdl.handle.net/10482/16939).

Fidalgo Castro, Alberto. 2012. "A religião em Timor-Leste a partir de uma perspectiva histórico-antropológica." Pp. 79-118 en Léxico Fataluco-Português, editado por Alberto Fidalgo Castro y Efrén Legaspi Bouza. Dili: Salesianos de Dom Bosco Timor-Leste. Última consulta: 15 de marzo de 2013. (http://hdl.handle.net/2183/9927).

Forbes, Henry Ogg. 1884. "On Some of the Tribes of the Island of Timor." The Journal of the Anthropological Institute of Great Britain and Ireland 13:402-30. Consulta: (http://links. jstor.org/sici?sici=0959-5295(1884)13<402:OSOTTO >2.0.CO;2-M).

Forman, Shepard. 1980. "Descent, Alliance, and Exchange Ideology among the Makassae of East Timor." Pp. 152-77 en The Flow of Life: Essays on Eastern Indonesia, editado por James J. Fox. Cambridge, Massachusetts y Londres, England: Harvard University Press.

Fox, James J. 1987. "The House As a Type of Social Organization on the Island of Roti." Pp. 215-24 en De la butte au palais: Sociétés "à maison" en Asie du Sud-Est insulaire, editado por Charles Macdonal. París: Éditions du Centre National de la Recherche Scientifique.

Fox, James J. 1994. "Reflections on 'Hierarchy' and 'Precedence." History and Anthropology 7(14):87-108.

Fox, James J. 2006. Inside Austronesian Houses. Perspectives on Domestic Designs for Living. Canberra: ANU E Press.

Gárate Castro, Luis Alberto. 2010. “As uma lulik e o contexto da sua localização no espaço.” en Património cultural de Timor-Leste. As uma lulik do distrito de Ainaro. Ferrol: Secretaría de Estado da Cultura da República Democrática de Timor-Leste.

Gárate Castro, Luis Alberto y Cecilia Assís. 2010. Património cultural de Timor-Leste. As uma lulik do distrito de Ainaro. Ferrol: Secretaría de Estado da Cultura da República Democrática de Timor-Leste.

García Canclini, Néstor. 2001. "El porvenir del pasado.” Pp. 157-94 en Culturas Híbridas. Estrategias para entrar y salir de la modernidad. Buenos Aires, Barcelona, México: Paidós.

Gillespie, Susan D. 2000. "Lévi-Strauss. Maison and Société À Maisons." Pp. 21-52 en Beyond Kinship. Social and Material Reproduction in House Societies, editado por Rosemary A. Joyce y Susan D. Gillespie. Philadelphia: Pennsylvania Press.

Gomes, Francisco de Azevedo. 1972. “Os fataluku.” B.A. dissertation, , Instituto Superior de Ciências Sociais e Política Ultramarina, Universidade Técnica de Lisboa, Lisboa.

Graham, Penelope. 1994. "Rhetorics of Consensus, Politics of Diversity:: Church, State and Local Identity in Eastern Indonesia." Social Analysis: The International Journal of Cultural E Social Practice 34:122-43. 
Gunter, Janet. 2007. “Communal Conflict in Viqueque and the 'Charged' History of'59." The Asia Pacific Journal of Anthropology 8(1):27-41. Ultima consulta: 29 de noviembre de 2007. (http://dx.doi.org/10.1080/14442210601177977).

Gusman, Primus, Tri Priantoro, Aloisius Poleng, Eusebio da Costa, y Paulus Susilo. 1991. Pengobatan Tradisional Di Timor Timur. Dili: Proyek Inventarisasi dan Pembinaan NilaiNilai Budaya Daerah Istimewa Yogyakarta, Departemen Pendidikan dan Kebudayaan.

Henley, David y Jamie S. Davidson. 2007a. "In the Name of Adat: Regional Perspectives on Reform, Tradition, and Democracy in Indonesia." Modern Asian Studies 42(04):815-52. Última consulta: 31 de agosto de 2014. (http://www.journals.cambridge.org/abstract_ S0026749X07003083).

Henley, David y Jamie S. Davidson. 2007b. "Introduction Radical Conservatism - the Protean Politics of Adat." Pp. 1-49 en The Revival of Tradition in Indonesian Politics. The deployment of adat from colonialism to indigenism, editado por Jamie S. Davidson y David Henley. Oxon: Routledge.

Hicks, David. 2004. Tetum Ghost $\mathcal{E}^{2}$ Kin. Fertility and Gender in East Timor. Long Grove, Illinois: Waveland Press, Inc.

Hicks, David. 2008. "Afterword. Glimpses of Alternatives-the Uma Lulik of East Timor." Social Analysis 52(1):166-80.

Hicks, David. 2011. “A pesquisa etnográfica no Timor Português.” Pp. 31-45 en Ita maun alin... O livro do irmão mais novo. Afinidades antropológicas em torno de Timor-Leste, editado por Kelly Cristiane da Silva y Lúcio Manuel Gomes de Sousa. Lisboa: Edições Colibri. A IELTsar se vai ao longe; 35.

Hicks, David. 2013. "Adat and the Nation-State: Opposition and Synthesis in Two Political Culturas." Pp. 25-43 en The Politics of Timor-Leste. Democratic Consolidation after Intervention, editado por Michael Leach y Damien Kingsbury. New York: Southeast Asia Program, Cornell University.

Hidayah, Zulyani et al. 1990. Upacara Tradisional Daerah Timor Timur. Jakarta: Departemen Pendidikan dan Kebudayaan, Direktorat Jenderal Kebudayaan, Direktorat Sejarah dan Nilai Tradisional, Proyek Inventarisasi dan Pembinaan Nilai-Nilai Budaya.

Joyce, Rosemary A. y Susan D. Gillespie. 2000. Beyond Kinship. Social and Material Reproduction in House Societies. Philadelphia: University of Pennsylvania Press.

Keane, Webb. 1995. “The Spoken House: Text, Act, and Object in Eastern Indonesia." American Ethnologist 22(1):102-24. Consulta: (http://www.jstor.org/stable/646048).

Kovar, Annika y Andrew Harri-ngton. 2013. Breaking the Cycle of Domestic Violence in TimorLeste. Acces to Justice Opinios, Barriers, and Decision Making Processes in the Context of Legal Pluralism. Dili. Última consulta: 10 de octubre de 2013 (http://www.tl.undp.org/content/ dam/timorleste/docs/reports/DG/Domestic Violence Report_with cover FINAL.pdf).

Lameiras-Campagnolo, Maria Olímpia. 1975. "L'habitation des fataluku de Lórehe (Timor Portugais).” PhD dissertation, École Pratique des Hautes Études, Université René Descartes, Sciences Humaines Sorbonne, París.

Leach, Michael. 2012. "Longitudinal Change in East Timorese Tertiary Student Attitudes to National Identity and Nation Building, 2002-2010." Bijdragen tot de Taal-, Land-en Volkenkunde 168(2):219-52.

Lencastre, Júlio Garcês de. 1931. "O povo timorense. Seu aproveitamento na valorisação da colónia." Boletim da Sociedade de Geografia de Lisboa 49(3-4):55-72.

Levang, Patrice. 1997. La Terre D’en Face. La transmigration en Indonésie. París: Éditions de l'Orstom.

Lévi-Strauss, Claude. 1981. La vía de las Máscaras. 2a Edición. México: Siglo XXI Editores. 
Lombard-Jourdan, Anne. 1997. "François Péron et Charles Lesueur à Timor. Une chasse au crocodile en 1803." Archipel 54(1):81-121.

Lowenthal, David. 1998. El pasado es un país extraño. Madrid: Akal.

Macdonald, Charles. 1987. De la hutte au palais sociétés «á maison» en Asie du Sud-Est Insulaíre. París: Éditions du CNRS.

Magno, Costa y Antonio Coa. 2012. "Finding a New Path between Lisan and Democracy at the Suku Level." LOCAL-GLOBAL. Identity, Security, Community. Traversing customary community and modern nation-formation in Timor-Leste 11:166-78. Consulta: (http:// www.timor-leste.org/storage/13_Finding_a_new_path_between_lisan_democracy_at_ the_suku_level_J_da_CostaMagno_ACoa.pdf).

McWilliam, Andrew. 2005. "Houses of Resistance in East Timor: Structuring Sociality in the New Nation." Anthropological Forum 15(1):27-44. Última consulta: 21 de febrero de 2014. (http://www.tandfonline.com/doi/abs/10.1080/0066467042000336698).

Nixon, Rodney Stafford. 2012. Justice and Governance in East Timor. Indigenous Approaches and the "New Subsistence State." Oxon: Routledge.

Peel, J. D. Y. 1989. “The Cultural Work of Yoruba Ethnogenesis.” Pp. 198-215 en History and Ethnicity. ASA Monographs, 27, editado por Elizabeth Tonkin, Maryon McDonald, and Malcom Chapman.

Pena Castro, María Jesús. 2010a. "Las casas sagradas de Timor Oriental como estrategia de consolidación de la identidad nacional en un joven estado." Pp. 327-34 en Libro de actas del IV Congreso Internacional de Patrimonio Cultural y Cooperación al Desarrollo. 16, 17 y 18 de junio de 2010. Sevilla (España), editado por Cristina Ordaz Benet et al. http://www.iaph. es/export/sites/default/sites/patrimonioydesarrollo/LibroActas.pdf: Instituto Andaluz de Patrimonio Histórico. Última consulta: 28 de febrero de 2013. (http://digital.csic.es/bitstream/10261/25880/3/2010_Actas IV Congreso Internacional Patrimonio Cultural.pdf).

Pena Castro, María Jesús. 2010b. “Os agrupamentos ou conjuntos de uma lulik.” Pp. 125-35 en Património cultural de Timor-Leste. As uma lulik do distrito de Ainaro, editado por Luis Alberto Gárate Castro y Cecilia Asís. Ferrol: Secretaría de Estado da Cultura da República Democrática de Timor-Leste.

Picard, Michel. 1997. "Cultural Tourism, Nation-Building, and Regional Culture: The Making of a Balinese Identity." Pp. 181-214 en Tourism, Ethnicity and the State in Southeast Asia and the Pacific, editado por M. Picard y R. Wood. Honolulu: University of Hawai'i Press.

Prüller, Vanessa. 2008. "The 2006 Crisis in East Timor - an Ethnic Conflict?.”MA dissertation, Faculty of Philosophy, Southeast Asian Studies I, University of Passau. Consulta: (http:// www.iycdili.org/iyc_Docs/018_Prueller_2006_crisis_ET_ethnic_conflict.pdf).

RDTL. 2002. “Constituição Da República Democrática de Timor- Leste.”Última consulta: 12 de marzo de 2014. (http://timor-leste.gov.tl/wp-content/uploads/2010/03/Constituicao_ RDTL_PT.pdf).

RDTL. 2009. "Política Nacional Da Cultura.” Jornal da República I(41):3786-3801.

Renard-Clamagirand, Brigitte. 1975. "La maison Ema." Asie du Sud-Est et Monde Insulindien 2:35-60.

Renard-Clamagirand, Brigitte. 1982. Marobo, une société ema de Timor. París: Langues et Civilisation de L'Asie du Sud-Est et du Monde Insulindien 12, SELAF.

Roosens, Eugeen E. 1989. Creating Ethnicity: The Process of Ethnogenesis. Thousand Oaks: SAGE Publications.

Roque, Ricardo. 2011. "Etnografias coloniais, tecnologias miméticas: a administração colonial e os usos e costumes em Timor-Leste no final do século XIX.” Pp. 155-68 en Ita maun alin... O livro do irmão mais novo. Afinidades antropológicas em torno de Timor-Leste, editado 
por Kelly Cristiane da Silva y Lúcio Manuel Gomes de Sousa. Lisboa: Edições Colibri. A IELTsar se vai ao longe; 35 .

Schefold, Reimar, Gaudenz Domenig, y Peter Nas. 2004. Indonesian Houses. Tradition and Transformation in Vernacular Architecture. Singapore: Singapore University Press.

Schefold, Reimar, Gaudenz Domenig, y Peter Nas. 2008. Indonesian Houses. Volume 2. Survey of Vernacular Architecture in Western Indonesia. Leiden: KITLV Press.

Scott, James C. 1985. Weapons of the Weak: Everyday Forms of Peasant Resistance. New Haven y Londres: Yale University Press.

Silva, Kelly Cristiane da. 2012. As nações desunidas: Práticas da ONU e a estruturação do Estado em Timor-Leste. Belo Horizonte: Editora UFMG.

Silva, Kelly Cristiane da y Daniel Schroeter Simião. 2012. “Coping with 'traditions': The Analysis of East-Timorese Nation Building from the Perspective of a Certain Anthropology Made in Brazil." Vibrant 9(1):360-81. Ultima consulta: 20 de agosto de 2012. (http://www.vibrant.org.br/downloads/v9n1_silva_simiao.pdf).

Simião, Daniel Schroeter. 2005. "As donas da palavra. Gênero, justiça e a invenção da violência doméstica em Timor-Leste." $\mathrm{PhD}$ dissertation, Instituto de Ciências Sociais, Departamento de Antropologia, Programa de Pós-Graduação em Antropologia Social, Universidade de Brasília. Última consulta: 11 de marzo de 2014. (http://dan.unb.br/images/doc/Tese_062.pdf).

Simião, Daniel Schroeter. 2011. Sé Mak Sala Tenkeser Selu Sala. Brasil/Timor-Leste: IRIS DAN/UnB. Consulta: (http://www.youtube.com/watch?v=CyKnw2Vgz6M).

Simião, Daniel Schroeter. 2013. "Law, Justice and Compensation: Material and Moral Exchanges in East Timor Legal Practices." en Paper presented at 7th EuroSEAS Conference School of Social and Political Sciences -ISCSP. University of Lisbon-Lisbon, 02-05 July 2013.

Sousa, Lúcio Manuel Gomes de. 2007. "As casas e o mundo: identidade local e nação no património material/imaterial de Timor-Leste” editado por Fernando Cruz. Actas do III Congresso Internacional sobre Etnografia 196-227. Última consulta: 29 de agosto de 2012. (http://www.cultura.gov.tl/sites/default/files/LSousa_As_casas_e_o_mundo_identidade_local_2008.pdf).

Sousa, Lúcio Manuel Gomes de. 2010. "An tia: partilha ritual e organização social entre os Bunak de Lamak Hitu, Bobonaro, Timor-Leste" PhD dissertation, Doutoramento em Antropologia, Especialidade Antropologia Social, Universidade Aberta, Lisboa. Última consulta: 26 de junio de 2014. (http://repositorioaberto.uab.pt/handle/10400.2/1703)

Spyer, Patricia. 1996. "Diversity with a Difference: Adat and the New Order in Aru (Eastern Indonesia).” Cultural Anthropology 11(1):25-50.

Susilo, Paulus. 1991. Uma Fukun Di Timor Timur: Sumbangan Makalah Kongres Kebudayaan Tahun 1991. Dili: Kanwil Depdikbud Prop. Timor Timur.

Susilo, Paulus, Sukijo, Dara Windiyarti, y Ekanto Heru Subronto. 1997. Wujud, Arti, Dan Fungsi Puncak-Puncak Kebudayaan Lama Dan Asli Bagi Masyarakat Pendukungnya Di Daerah Timor Timur. Dili: Departemen Pendidikan dan Kebudayaan, Direktorat Jenderal Kebudayaan, Direktorat Sejarah dan Nilai-Nilai Tradisional, Bagian Proyek Pengkajian dan Pembinaan Nilai-Nilai Budaya Timor Timur.

Timor-Leste, Governo de. 2010. "Inauguração da casa sagrada do suco de Nunu Moge, no distrito de Ainaro." Última consulta: 4 de septiembre de 2014. (http://timor-leste.gov. $\mathrm{t} 1 /$ ?p=2690\&lang=pt).

TMII. 2012. "Museum Timor Timur."Última consulta: 4 de septiembre de 2014. (http://www. tamanmini.com/museum/museum-timor-timur-2?lang=en). 
Traube, Elizabeth G. 1980a. "Affines and the Dead: Mambai Rituals of Alliance.” Bijdragen tot de Taal-, Land-en Volkenkunde 136(1):90-115.

Traube, Elizabeth G. 1980b. "Mambai Rituals of Black and White." Pp. 290-314 en The Flow of Life: Essays on Eastern Indonesia, editado por James J. Fox. Cambridge, Massachusetts y Londres, England: Harvard University Press.

Traube, Elizabeth G. 1986. Cosmology and Social Life: Ritual Exchange among the Mambai of East Timor. Chicago y Londres: The University of Chicago Press.

Traube, Elizabeth G. 1989. "Obligations to the Source: Complementarity and Hierarchy in an Eastern Indonesian Society.” Pp. 321-44 en The Attraction of Opposites. Thought and Society in the Dualistic Mode, editado por David Maybury-Lewis y Uri Almagor. Ann Arbor. Michigan: The University of Michigan Press.

Traube, Elizabeth G. 1991. "Timorese Perspectives on Colonialism and Decolonization." en SSRC Workshop in East Timor. Washington, D. C. April 25, 1991. Consulta: (http://xdata. bookmarc.pt/cidac/t1/TL5888.pdf).

Trinidade, Jose "Josh." 2008. "An Ideal State for East Timor: Reconciling the Conflicting Paradigms." Última consulta: 7 de mayo de 2013. (http://www.cdu.edu.au/timorlesteconference/documents/trindade_ideal_state_for_East_Timor_digPAPER.pdf).

Vellinga, Marcel. 2004a. Constituting Unity and Difference: Vernacular Architecture in a Minangkabau Village. Leiden: KITLV Press.

Vellinga, Marcel. 2004b. "The Use of Houses in a Competition for Status. The Case of Abai Sangir (Minangkbau).” Pp. 155-76 en Indonesian Houses. Volume 1: Tradition and Transformation in vernacular architecture. Leiden y Singapur: KITLV Press \& Singapore University Press.

Waterson, Roxana. 2009. The Living House. An Anthropology of Architecture in South-East Asia. Tuttle Publishing.

Wilson, T. D. y A. Ypeij. 2012. “Tourism, Gender, and Ethnicity." Latin American Perspectives 39(6):5-16. Última consulta: 19 de agosto de 2014. (http://lap.sagepub.com/cgi/ doi/10.1177/0094582X12453896).

Windiyarti, Dara, Primus Gusman, Paulus Susilo, y Eusebio da Costa. 1993. Dampak Pengembangan Pariwisata Terhadap Kebidupan Sosial Di Daerah Timor Timur. Dili: Departemen Pendidikan dan Kebudayaan, Direktorat Jenderal Kebudayaan, Direktorat Sejarah dan Nilai Tradisional, Bagian Proyek Penelitian, Pengkajian dan Pembinaan NilaiNilai Dudaya [i.e. Budaya] Timor Timur.

World Bank. 2013. Road for Cultural Heritage: Policy Note on Cultural Heritage and Infrastructure Development in Timor-Leste. Washington DC. Consulta: (http://documents.worldbank. org/curated/en/2013/07/18103769/road-cultural-heritage-policy-note-cultural-heritage-infrastructure-development-timor-leste).

Wortelboer, Von Wilco. 1955. "Zur Sprache und Kultur der Belu (Timor).” Anthropos 50:153200.

Yampolsky, Philip. 1995. "Forces for Change in the Regional Performing Arts of Indonesia." Bijdragen tot de Taal-, Land-en Volkenkunde 151(4):700-725.

Yoder, Laura Suzanne Meitzner. 2007. "Hybridising Justice: State-Customary Interactions over Forest Crime and Punishment in Oecusse, East Timor." The Asia Pacific Journal of Anthropology 8(1):43-57. Última consulta: 1 de marzo de 2007. (http://dx.doi. org/10.1080/14442210601161732). 


\section{AwKWARd ObJects: the place of Uma-LULik In Timor-Leste's Nation-State}

This paper aims to contribute to current knowledge of Timor-Leste's national building, specifically on the effects of cultural policies related to the "uma-lulik" (sacred houses). I argue that the aestheticization process of Timorese culture, specially prompted by heritage-oriented interventions, results in the lost of the meaning of culture as a living social practice. This reductionist approach, which links culture to its most visible elements, responds to a cosmetic usage. Throughout this re-semanticization process, culture loses its raison d'etre and is turned into a source of tensions and potential conflicts.

Keywords: Timor-Leste, national building, house societies, cultural policies, aestheticization.

Data recibido: 2014-09-09

Data aceptado: 2015-01-18 